\title{
Endolaryngeal Excision of Recurrent Laryngeal Nerve Neurofibroma
}

\author{
${ }^{1}$ Inita Matta, ${ }^{2}$ Sunita Chhapola Shukla, ${ }^{3}$ Prabodh Karnik
}

\begin{abstract}
Vagal schwannomas, compromising $1 \%$ of head and neck neoplasms, are the commonest neurogenic tumors of the neck. Recurrent laryngeal nerve neurofibromas are even rarer. We report such a case, excised totally by an endolaryngeal approach. New trends in diagnosis and immunohistochemistry are discussed.
\end{abstract}

Keywords: Calretinin, CD 34, CD 56, Endolaryngeal surgery, Factor XIII a, Ki-67, Neurofibroma, Recurrent laryngeal nerve, S-100 protein, Schwannoma.

How to cite this article: Matta I, Shukla SC, Karnik P. Endolaryngeal Excision of Recurrent Laryngeal Nerve Neurofibroma. Int J Phonosurg Laryngol 2015;5(1):35-38.

\section{Source of support: Nil}

Conflict of interest: None

\section{INTRODUCTION}

Laryngeal nerve sheath tumors do not have a common association with neurofibromatosis though they are histologically similar. ${ }^{1}$ Isolated recurrent laryngeal nerve neurofibromas are rare tumors and excision via an 'open neck' approach is the advocated method of treatment.

Currently, the endolarygeal approach is fast gaining popularity for excision of laryngeal tumors. The excellent visual control offered by the operating microscope, coupled with Hopkins rod telescopes facilitates the endolaryngeal approach. The avoidance of a neck scar is an added advantage. Preoperative assessment of the limits of the tumor by computed tomography (CT) and magnetic resonance imaging (MRI) is invaluable in deciding the feasibility of excision through a limited approach. The benign nature of the neurofibroma would be indicated by a preoperative fine needle aspiration cytology showing an absence of mitotic figures.

Neurofibromas of peripheral cranial nerves (when not part of multiple neurofibromas) are usually single, circumscribed masses and exhibit spindle cells without atypia. Mitosis is common in schwannomas while

${ }^{1}$ Senior Dy. CMO, ${ }^{2}$ Senior Surgeon, ${ }^{3}$ Honorary Surgeon

${ }^{1-3}$ Department of ENT, Mumbai Port Trust Hospital, Mumbai Maharashtra, India

Corresponding Author: Sunita Chhapola Shukla, Senior Surgeon, Department of ENT, Flat No. 4, Charak, Mumbai Port Trust Hospital, Campus, Mumbai, Maharashtra, India, Phone: 919869256377, e-mail: drsunita7ent@yahoo.co.in mitotic activity in aneurofibromas may be indicative of a malignancy. ${ }^{2}$ Neurofibromas of the recurrent laryngeal nerve may present with unilateral vocal cord palsy. ${ }^{3}$ The denervated cord may be managed subsequently by a medialisation procedure.

\section{CASE REPORT}

A 23-year-old female presented with progressively increasing hoarseness of voice since 3 months. There was no dysphagia or dyspnoea. Flexible fibreoptic laryngoscopy showed a diffuse swelling in the region of the left supraglottic area with non-visualization of the ipsilateral cord. An ultrasonography (USG) of the neck revealed a homogenous, hypoechoic mass in the tracheoesophageal groove. It measured $3.9 \mathrm{~cm}$ (superior-inferior), $1.4 \mathrm{~cm}$ (anterior-posterior) and $2.5 \mathrm{~cm}$ (transverse) and was possibly neurogenic, arising from the left recurrent laryngeal nerve. Subsequently MRI with contrast substantiated this. The well-circumscribed mass lay at the level of the anterior commissure, extending into the left paratracheal soft tissue, indenting the postcricoid esophagus posteriorly (Fig. 1). Its caudal limit was the lower pole of the left thyroid lobe. Ultrasonography-guided fine needle aspiration cytology (FNAC) yielded spindle cells with no atypia. There were no other findings locally or systemically.

Considering the young, unmarried status of the patient, it was decided to attempt an endolaryngeal microscopic removal, thus avoiding a neck scar. Consent for open neck surgery was also obtained in case problems were encountered in the limited approach. The larynx was exposed in the usual way for microlaryngoscopy. An incision was made in the overlying mucosa of the mass (Fig. 2) and gentle dissection was used to separate the mass from its surrounding tissue (Fig. 3). Bipolar cautery was used for hemostasis. The mass was removed completely (Fig. 4). There was no problem, respiratory or otherwise, encountered in the postoperative period. Subsequent follow-up examination by fibreoptic flexible laryngoscopy showed a healthy, healing mucosa in the left supraglottic region. Postoperatively, the left vocal cord was paralysed. The histopathology of the excised specimen was reported as a cellular spindle cell tumor in a fascicular pattern in a myxoid background with no mitotic activity (Fig. 5). The immunohistochemistry of the specimen tested positive for S-100 protein and CD 34 . 


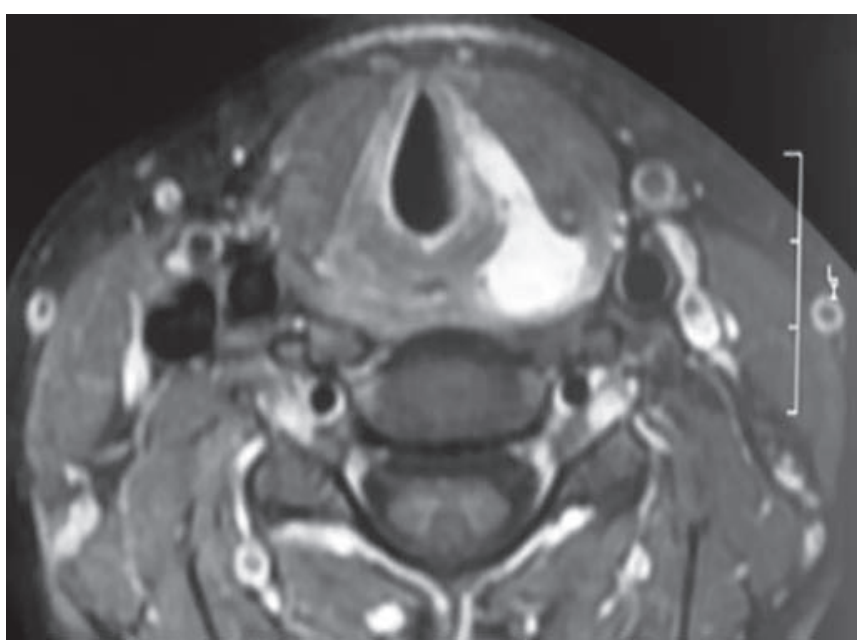

Fig. 1: Magnetic resonance imaging showing well-circumscribed mass lay at the level of the anterior commissure, extending into the left paratracheal soft tissue, indenting the postcricoid esophagus posteriorly

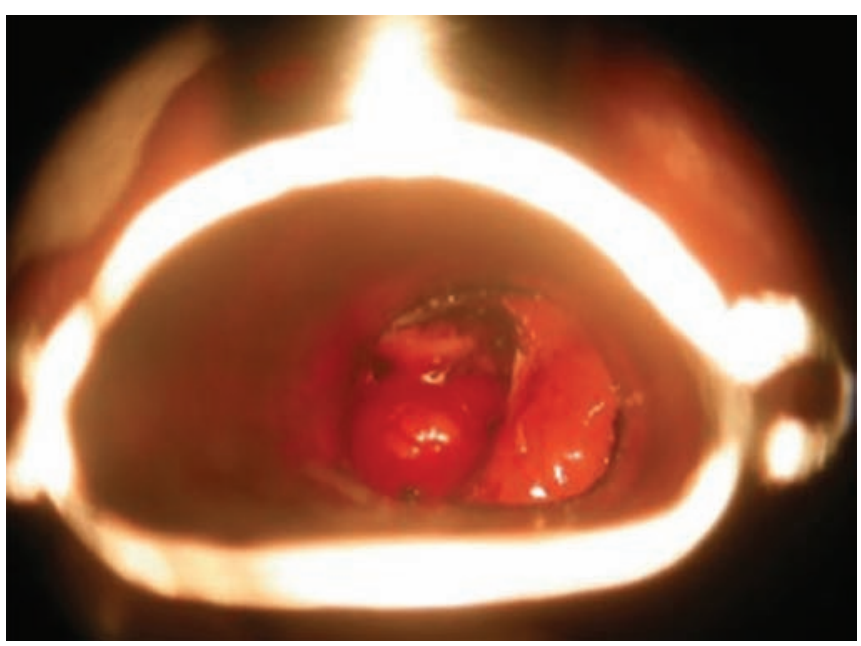

Fig. 3: Dissection of mass

Medialization was done for the left vocal cord at a later date. On follow-up till 2 years postoperative period, there was no recurrence of growth as checked by MRI.

\section{DISCUSSION}

Most neurogenic tumors in the neck are vagal schwannomas, comprising $1 \%$ of head and neck neoplasms. ${ }^{4}$ Isolated neurofibromas (not forming part of a neurofibromatous state) are even rarer. ${ }^{5}$ The neurofibromas originate from the schwann cell and the perineurium. Thus, it is inseparable from the nerve it arises from. Excision of the lesion necessarily sacrifices the nerve, and secondary rehabilitation of the larynx is required, if the neurofibromas arise from the recurrent laryngeal nerve. In general, sporadic neurofibromas are histologically indentical to those seen in neurofibromatosis type $1 .^{6}$ Schwannomas arise solely from schwann cells and are usually separable from the nerve.

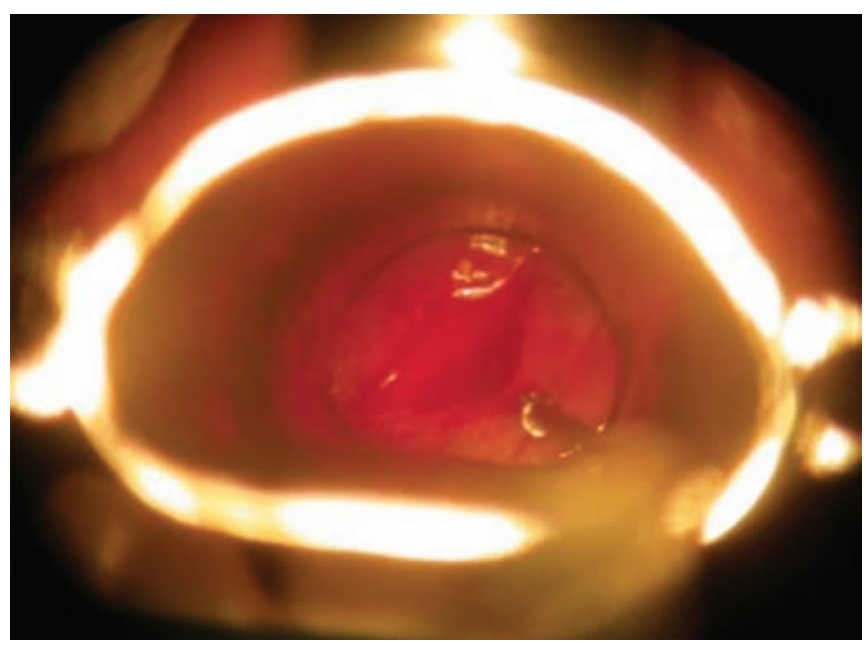

Fig. 2: Incision on the mucosa overlying the mass

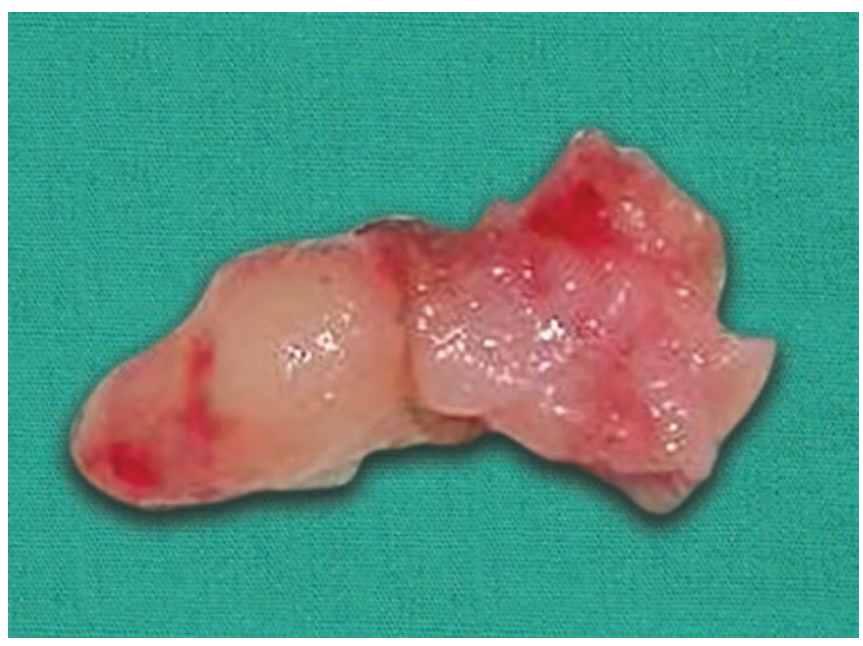

Fig. 4: The excised mass

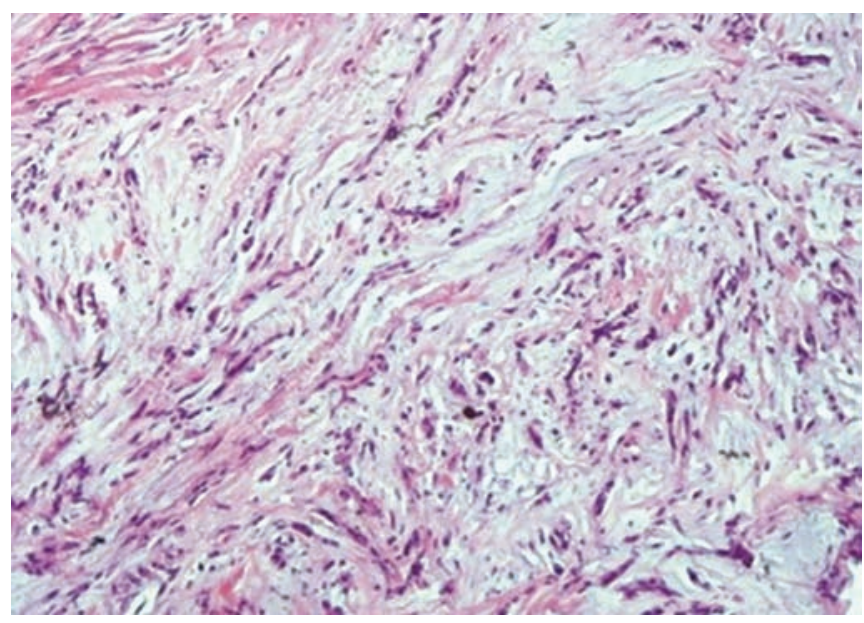

Fig. 5: Histopathology, showing a cellular spindle cell tumor in a fascicular pattern in a myxoid background with no mitotic activity

Neurofibromas are characterized by slow growth and lack of pain..$^{7-9}$ A neurofibroma arising singly from a peripheral cranial nerve is usually an unencapsulated, 
fusiform swelling. Microscopically, they are loose, interlacing bands of delicate spindles cells, with a mixture of schwann cells, perineural cells and endoneural fibroblasts. They do not exhibit unusual mitotic activity or nuclear atypia. Evidence of these would indicate a malignant transformation. It needs to be differentiated from a schwannoma and a malignant fibrosarcomatous transformation. ${ }^{4,10}$ Neurofibromas are classified into major and minor varieties. Plexiform, diffuse and pacinianneurofibromas fall into the 'major' group while epitheloid, myxoid, glandular, xanothomatised, cellular and other neurofibromas fall into 'minor' group. ${ }^{11-13}$

The immunohistochemistry of nerve masses has provided a new insight into the characteristics mitosis of neurofibromas and their differentiation from schwannomas. In a classical presentation, hematoxylin and eosin staining may be sufficient to recognise neurofibromas. ${ }^{14}$ However, sometimes immunochemistry is needed to show-up the differences between neurofibroma and schwannomas.

S-100 protein is expressed by both neurofibromas and schwannomas, though the latter express it more strongly. ${ }^{14}$ Neurofibroma often demonstrates variable degree of S-100 immunoreactivity. ${ }^{15}$ Cells with S-100 protein resemble Schwann cells ultrastructurally. Neurofibromas consist of Schwann cells with S-100 protein and also contain cells without S-100 protein, namely perineurial, intermediate cells and endoneurial fibroblasts. ${ }^{16}$

CD 34 positive stromal cells are typically seen in neurofibromas. ${ }^{17,18}$ Calretinin was cited as an aid to distinguish schwannomas from neurofibromas by Fine et al. ${ }^{19}$ Calretinin is strongly expressed by schwannomas. ${ }^{18}$ Neurofibromas are positive for factor XIIIa and negative for CD 56. ${ }^{20,21}$ The presence of neurofilament within the tumor would be indicative of neurofibromas as they grow within the nerve of origin in contrast to schwannomas. ${ }^{14}$ If the question of malignancy arises in a tumor of this kind, Ki-67 would aid the diagnosis as it has a low proliferative fraction in benign lesions. ${ }^{14}$ However, some tumors of a hybrid nature may defy attempts at differentiation despite the battery of immunohistochemistry tests available.

The imaging of nerve masses offers up a choice of ultrasound, CT and MRI. Ultrasonography is a simple, non-invasive tool to determine the site, nature and extent of neck swellings. A recurrent laryngeal nerve neurofibroma shows up as a circumscribed lesion. A taper of the mass at the inferior end would indicate its association with the nerve of origin. ${ }^{22}$ Computed tomography also reveals a recurrent laryngeal nerve neurofibroma to be a well-circumscribed mass in the paratracheal space while an MRI may often not be able to differentiate a solitary neurofibroma from a schwannoma. ${ }^{23}$
It is noteworthy that in our patient, the diagnosis was made reliably on ultrasound and FNAC.

\section{CONCLUSION}

Recurrent laryngeal nerve neurofibromas are rare tumors and the treatment is excision of the mass. Ultrasonography and FNAC are reliable diagnostic tools. Since recurrent laryngeal nerve neurofibromas cannot be dissected off the originating nerve, their removal would necessarily lead to a postoperative paralysis of the nerve. An endolaryngeal approach, if feasible is advocated as it avoids a neck scar. The resulting vocal cord palsy may be treated by a medialisation procedure.

\section{REFERENCES}

1. Cihangiroglua M, Yilmaze S, Topsalkab C, et al. Laryngeal Neurofibroma Asssociated with Neurofibromatosis Type 2.

2. Solitary Neurofibroma. Available at: http://www.histopathology-india.net/Neurofibroma.htm.

3. Rees G. Neurofibroma of the recurrent laryngeal nerve. Chest 1971;60:414-418.

4. Stell and Maran's Head and Neck Surgery: Benign neck disease. 4th ed. chapter 11, p. 193.

5. Contant A, Acquaviva F, Peraldi R, Fanton Y. Neurofibroma of recurrent nerve: apropos of case (Article in French) Rev Laryngol Otol Rhinol (Bord) 1995;116(3):225-228.

6. Weiss SW, Goldman JR. Neurofibroma, In: Enzinger and Weiss's soft tissue tumors. 4th ed. Edited by Weiss SW, Goldman JR. St Louis, London, Philadelphia, Sydney, Toronto: Mosby; 2001:1122-1146.

7. Dickersin GR. The electron microscopic spectrum of nerve sheath tumors. Ultrastruct Pathol 1987;11:103-146.

8. Erlandson RA, Woodruff JM. Peripheral nerve sheath tumors: an electron microscopic study of 43 cases. Cancer 1982;49: 273-287.

9. Peltonen J, Jaakkola S, Virtanen I, Pelliniemi L. Perineurial cells in culture: an immunocytochemical and electron microscopic study. Lab Invest 1987;57:480-488.

10. Chang Lo M. Laryngeal involvement in von Recklinghausen's disease: a case report and review of the literature. Laryngoscope 1977;87:435-442.

11. MacDonald AW, Fletcher A. Expression of cytokeratin in the epithelium of dentigerous cysts and odontogenic kerotocysts: an aid to diagnosis. J Clin Pathol 1989;42:736-739.

12. Megahed M. Histopathological variants of neurofibroma: a study of 114 lesions. Am J Dermatopathol 1994;16:486-495.

13. Shaktawat SS, Golka D. Floret-like multinucleated giant cells in neurofibroma. Diagn Pathol 2007;2:47.

14. Miller RT, ProPath. The Focus-Immunohistochemistry www. propath.com Oct 2004.

15. Stefansson K, et al. S-100 protien in soft tissue tumors derived from Schwann cells and melanocytes. Am J Pathol 1982;106:261.

16. Hirose T, Sano T, Hizawa K. Ultrastructural localization of S-100 protien in neurofibroma. Acta Neuropathologica 69(1-2): 103-110. 
17. Hirose $\mathrm{T}$, et al. Immunohistochemical demonstration of EMA/Glut-1-positive perineurial cells and CD 34-positive fibroblastic cells in peripheral nerve sheath tumors. Mod Pathol 2003 Apr;16(4):293-298.

18. Chaubal A, Paetau A, Zoltick P, Miettinen M. CD 34 immuno reactivity in nervous system tumors. Acta Neuropathol 1994; 88:454-458.

19. Fine SW, McClain SA, Li M. Immunohistochemical staining for calretinin is useful for demonstrating schwannomas from neurofibromas. Am J Clin Pathol 2004 Oct;122: 552-559.
20. Gray $\mathrm{MH}$, et al. Immunohistochemical demonstration of factor XIIIa expression in neurofibromas. A practical means of differentiating these tumors from neurotized nevi and schwannomas. Arch Dermatol 1990 Apr;126(4):472-476.

21. Milliard CL, et al. Neural cell adhesion molecule (CD 56) expression in mesenchymal tumors. Mod Pathol 2003 Jan; 14(1):16A (abstract 72).

22. Robert A Sofferman. Interpretation of ultrasonography. The Otolaryngologic Clinics of North America 2010 Dec;43(6):1193.

23. Stell and Maran's Head and Neck Surgery: Radiology. 4th ed. chapter 3, p. 44. 